\title{
A PERCEPÇÃO DO ENFERMEIRO SOBRE CUIDADOS A PACIENTES ONCOLÓGICOS
}

ZUCOLO, Fernanda; PAULINO, Camila Pereira. Bacharéis em Enfermagem pelo Centro Universitário de Araraquara - UNIARA. E-mail: fer_zucolo@hotmail.com; ca.pereira25@ hotmail.com.

WHITAKER, Maria Carolina Ortiz. Enfermeira. Doutora em Ciências. Profa. Assistente do Curso de Enfermagem do Centro Universitário de Araraquara - UNIARA. E-mail: carolwhitaker97@gmail.com.

\begin{abstract}
Resumo
O câncer se caracteriza como uma doença que acomete diferentes etnias, sexo e nível socioeconômico.A incidência de câncer está cada vez maior, e, segundo estimativas nacionais e internacionais, os números continuarão aumentando. Diante dessa constatação, o enfermeiro deve estar preparado para desenvolver um plano de cuidados a pacientes oncológicos atendendo às necessidades biológicas, sociais e emocionais decorrentes do diagnóstico, tratamento e sobrevivência. O estudo tempor objetivo conhecer a percepção do enfermeiro acerca do cuidar de enfermagem ao paciente com câncer. É um estudo de natureza descritiva com abordagem metodológica qualitativa dos dados, realizado com 12 enfermeiros que desenvolviam atividades assistenciais a pacientes com câncer de um hospital escola do interior do estado de São Paulo. Os dados foram coletados com base em uma entrevista semiestruturada. Os dados foram organizados ao redor de dois temas principais: a percepção e as dimensões do cuidar e os cuidados ao paciente oncológico. Os resultados revelaram que, para os enfermeiros, a assistência é direcionada a questões como os cuidados durante o tratamento, administração de quimioterápicos, alívio da dor e as dimensões emocionais da convivência ao paciente com câncer, no processo de terminalidade e morte. Concluímos que o cuidar do paciente oncológico requer conhecimento especializado e humanizado de toda a equipe multiprofissional envolvida neste processo.
\end{abstract}

Palavras-chave: Cuidar; Oncologia; Enfermagem.

\section{NURSE'S PERCEPTION OF ONCOLOGICAL PATIENTS' CARE}

\begin{abstract}
Cancer is characterized as a disease that affects different ethnicities, gender and socioeconomic status. The incidence of cancer is increasing, and, according to national and international estimates, the numbers will continue increasing. Based on this finding, the nurse must be prepared to develop a care plan for cancer patients according to the biological, social and emotional needs resulting from the diagnosis, treatment and survival. The study aims to know the nurse's perception about the nursing care to the patient with cancer. It is a descriptive exploratory study with qualitative methodological approach of data carried out with twelve nurses who developed assistance activities with patients with cancer of a school hospital in the State of São Paulo, Brazil. The data were collected using a semi-structured interview. The data were organized around two main themes: the perception and the dimensions of the care and the oncology patient care. The results revealed that, for nurses, assistance is directed to issues such as the care during treatment, administration of chemotherapy, pain relief and emotional dimensions of patient interaction with cancer, in terminality process and death. We conclude that the oncological patient care requires specialized and humanized knowledge of the entire multidisciplinary team involved in this process.
\end{abstract}

KeYwords: Care; Oncology; Nursing. 


\section{INTRODUÇão}

Compreendemos que o cuidado humano é indispensável nas diversas situações na vida de uma pessoa, desde a promoção da saúde, na prevenção de doenças, no decorrer de enfermidades e seus agravos, nas incapacidades e até durante o processo de morrer (CELICH, 2003).

A enfermagem, enquanto ciência, traz o cuidado alicerçado nas bases científicas, onde a assistência se torna sistematizada, gerando assim um verdadeiro e contínuo processo de atendimento a seus pacientes (TANNURE; GONÇALVES, 2008).

Atualmente, temos fundamentado o cuidar de enfermagem por meio do Processo de Enfermagem, a qual torna possível a operacionalização do trabalho de enfermagem, dividido didaticamente em cinco fases, sendo estas a coleta de dados, onde será colhido todo o histórico e informações que nos levem a diagnósticos e causas, e assim intervenções mais precisas, e também o exame físico; diagnóstico de enfermagem; planejamento dos cuidados que serão realizados; implementação dessas intervenções; e avaliação como forma de confirmar se a assistência prestada foi a mais correta e eficaz, ou se precisamos realizar outras intervenções (BRASIL, 2009).

Considerando que a atuação do enfermeiro se dá em diferentes momentos da vida do ser humano, e deve ser desenvolvido para atender às necessidades do cliente, da família e da comunidade, conhecer a percepção dos profissionais sobre o cuidar é importante, ainda mais ao considerarmos o cuidado a indivíduos acometidos por neoplasias malignas, uma doença de repercussão biopsicossocial desde o seu diagnóstico até o seu processo de cura ou terminalidade de vida.

As neoplasias malignas são a segunda causa de morte por doença no Brasil, representando $17 \%$ dos óbitos de causa conhecida. No Brasil, as estimativas para o ano de 2012 serão válidas também para o ano de 2013, e apontam a ocorrência de aproximadamente 518.510 casos novos de câncer, incluindo os casos de pele não melanoma, reforçando a magnitude do problema do câncer no país. Extraindo os casos de câncer da pele não melanoma, estima-se um total de 385 mil casos novos (BRASIL, 2012).

Uma possível explicação desse grande percentual são as mudanças nos hábitos de vida da população, em relação, por exemplo, à alimentação e ritmo de vida (sedentarismo), o que a expõe a um maior número de fatores de risco (BRASIL, 2009).

Uma pesquisa sobre a visão de outros profissionais da saúde a respeito do papel da enfermagem na equipe multiprofissional ressalta a importância dessa atuação, não apenas na área prática do cuidado, mas também na sua parte administrativa. Este estudo referenciado também traz como função do enfermeiro na equipe de saúde a liderança, sendo importante sua forma de posicionar-se abertamente, além de seu conhecimento técnico e humanização nas relações interpessoais (BACKES et al., 2008).

Dessa forma, profissionais da saúde buscam aumentar os seus conhecimentos combases científicas, para melhora no campo de atuação prática aos cuidados dessa clientela. Estudos demonstram o interesse de enfermeiros em atualizar os seus conhecimentos na área da oncologia, e dessa forma efetivar a qualidade da assistência prestada (SILVERA, 2006).

Os cuidados de enfermagem ao paciente oncológico devem abranger os aspectos biológicos, emocionais e sociais da enfermidade. É necessário também deter conhecimento em relação às terapias antineoplásicas, sua administração, os seus efeitos colaterais e a manutenção de dispositivos venosos de longa permanência. Isso demanda tempo e dedicação para ser adquirido. Esse conhecimento faz com que o profissional aja com segurança ao tratar do paciente oncológico, diante das diversas situações que podem ocorrer (FONTES; ALVIM, 2007).

Ao considerar as neoplasias como problema de saúde pública, devido a seus índices de morbidade e mortalidade, entende-se que os profissionais de saúde devam estar capacitados para prestar atendimento a essa população de ordem preventiva, curativa e paliativa. $\mathrm{O}$ cuidado ao paciente oncológico deve ser planejado com valorização às necessidades apresentadas por essa clientela. Assim, conhecer a 
percepção, a vivência de enfermeiros em um hospital geral, que presta assistência a pacientes oncológicos, pode ser uminstrumento para identificarmos como têm sido os cuidados de enfermagem para essa clientela nos serviços terciários de saúde.

\section{OBjetivos}

O presente trabalho tem por objetivos conhecer a percepção do enfermeiro acerca do cuidar de enfermagem ao paciente com câncer. Descrever as dimensões do cuidar para os enfermeiros e identificar cuidados realizados por enfermeiros, específicos aos pacientes oncológicos.

\section{Metodologia}

O presente estudo é uma pesquisa de campo, de natureza descritiva, com abordagem metodológica qualitativa dos dados. $\mathrm{O}$ estudo foi desenvolvido em um hospital-escola, classificado como uma unidade de nível terciário localizado em município no interior do estado de São Paulo.

Para este estudo, consideramos o referencial de Backes et al. (2008), que descreve um enfermeiro assistencial como o profissional que presta cuidado direito ao paciente, avaliando as suas necessidades, a fim de proporcionar a sua recuperação, realizando promoção em saúde.

Aceitaram participar do estudo um total de 12 enfermeiros, dentre os turnos da manhã, tarde e noite.

Durante a análise dos dados, utilizamos nomes fictícios, a fim de preservar a privacidade dos sujeitos. Depois classificamos os entrevistados mediante critérios como sexo, ano de conclusão da graduação e realização de curso de pós graduação.

Para explorar o campo de investigação, utilizou-se a entrevista semiestruturada. A entrevista foi realizada na sala de reuniões do setor pelo qual o enfermeiro era responsável, respeitando, assim, a privacidade do entrevistado. Os dados foram colhidos através de uma entrevista semiestruturada, com questões norteadoras, como: O que é "cuidar" para você? Como é para você cuidar de um paciente oncológico? Você identifica ou realiza algum cuidado específico aos pacientes com câncer? Foi feito uso de aparelho mp3 player para gravação da entrevista.

Em respeito à Resolução 466/12 do Conselho Nacional de Saúde, o projeto de pesquisa foi submetido à apreciação do Comitê de Ética em Pesquisa do Centro Universitário de Araraquara - UNIARA. Como parte da documentação prevista na legislação, utilizamos o Termo de Consentimento Livre e Esclarecido.

Iniciamos essa etapa partindo da ordenação dos dados mediante a transcrição integral do material. Na sequência realizamos a leitura do material empírico, procurando organizá-lo em agrupamentos descritivos, derivados do conteúdo manifesto e latente dos dados. Após essa fase, passamos para a classificação dos dados, realizada a partir da leitura exaustiva e repetida do material organizado em códigos, buscando identificar as ideias centrais e os aspectos relevantes existentes. Então, construímos dois temas centrais, a percepção e as dimensões do cuidar e os cuidados ao paciente oncológico; a partir deles, buscamos embasamento científico para traçar os resultados.

\section{Resultados}

\section{A percepção e as dimensões do cuidar}

$\mathrm{O}$ cuidar em enfermagem busca reconhecer e auxiliar as necessidades do ser cuidado e promover a saúde com olhar na dimensão biopsicossocial. Por meio de suas atividades, o enfermeiro busca conhecer as necessidades do indivíduo com olhar ampliado às necessidades provocadas pela doença; essas dimensões foram pontuadas pelos participantes, descrevendo suas percepções e experiências acerca do cuidar ao paciente com câncer. Para o grupo estudado, as dimensões do cuidado se revelam no cuidado humanizado, na atenção integral e no cuidado à família.

Ao analisarmos as entrevistas, observamos que algumas enfermeiras relataram que cuidar significa acolher, dar atenção, conversar e estar disponível para ouvir as queixas de cada um.

\footnotetext{
"...principalmente acolher, apoiar esse
} 
paciente... ele precisa de muita atenção, independente da patologia." (Ana)

"...tem que ter paciência, tem que se colocar no lugar do paciente, porque geralmente são pacientes muito carentes." (Daniela)

Para Vila (2006), humanizar o cuidado é acrescentar uma dose de carinho na técnica, alegrar o ambiente com iluminação e cores, manter cuidado individualizado, agregar a família ao cuidado, mantendo-a informada e com participação ativa durante o tratamento. O cuidado humanizado deve atender o paciente e sua família de modo integral. A integralidade, um dos princípios do SUS, é definida por Machado et al. (2007) como uma forma de cuidar com abordagem completa e holística, através do uso de tecnologias de saúde disponíveis a fim de prolongar a sobrevida do cliente.

Nesse sentido, os participantes do estudo revelaram suas percepções do cuidar ao paciente oncológico de forma integral como uma atitude intrínseca ao cuidado.

"Cuidar é você fazer tudo pelo paciente... é você ver o paciente num todo." (Estela)

"Cuidar pra mim é ver o paciente como um todo." (Fábia)

"Bom, o termo cuidar, ele é muito amplo, principalmente, assim, na parte da enfermagem, o cuidado vai desde um banho, uma medicação, um curativo... Eu acredito que o enfermeiro, ele cuida também da parte social desse paciente, da família, das necessidades que ele tem, oferece apoio psicológico..." (Laís)

Ampliando o olhar da percepção dos participantes ao cuidar do paciente oncológico, identificamos a descrição da necessidade do cuidado extensivo à família, principalmente em relação ao apoio emocional e ao esclarecimento sobre tratamento e doença.

"...é um cuidado ainda mais intenso, porque você tem que se preocupar com a família. Pois são mães que perderão o filho... Temos que cuidar da esposa, que está perdendo o marido... Precisamos cuidar, porque eles ficam aflitos, porque a qualquer momento o paciente vai embora. É preciso dar atenção, amparo ao familiar e cuidar de modo que a família sinta segurança." (Bruna)

"...Eu acho que todo mundo tem que ver a questão da família, porque a família que ela tem que... elas não aceitam, não querem perder, elas não querem acreditar..." (Estela)

Carvalho (2008) relata que o impacto de uma doença como o câncer afeta de forma intensa toda a família do paciente, impondo mudanças bruscas na rotina e dinâmica familiar. A doença causa grande sofrimento, e a precariedade das condições sociais, econômicas e culturais dos acometidos aumentam a vulnerabilidade social que o câncer impõe. Segundo Venâncio (2004), o apoio a esse tipo de paciente e sua família contribui no enfrentamento das dificuldades do tratamento, o que ajuda a desenvolver estratégias para o enfrentamento de situações estressantes causadas pela doença.

\section{Os cuidados ao paciente oncológico}

Este tema descreve sobre as experiências dos enfermeiros junto aos pacientes oncológicos, segundo as especificidades dos cuidados mediante ao tratamento, administração de quimioterápicos, alívio e controle da dor. As dimensões emocionais da convivência ao paciente com câncer, no processo de terminalidade, morte e o desgaste emocional do profissional frente ao cuidado complexo, requerido pelo paciente oncológico e sua família, também foram abordados neste tema.

Desde o diagnóstico da doença o paciente é submetido a diversos procedimentos, a fim de identificar a doença e sua classificação. $\mathrm{O}$ tratamento de escolha procura promover sobrevida prolongada e conter o crescimento das células malignas ou aliviar os sintomas associado ao tumor. Entre as modalidades terapêuticas mais utilizadas existem a cirurgia, a radioterapia e quimioterapia (BARBOSA; TELLES FILHO, 2008). 
A percepção do enfermeiro sobre cuidados...

Ao investigarmos sobre os cuidados específicos durante o cuidado ao paciente oncológico a atenção na administração de quimioterápicos, revelou-se como uma ação complexa e privativa do enfermeiro.

"...Às vezes o pessoal tem essa visão. Pensam que é um soro comum, não é um soro comum, não é uma medicação comum, é um quimioterápico..." (Fabia)

"...Aqui eu tenho pacientes que tomam quimioterapia, então eu tenho que me certificar que esse paciente precisa de um controle mais rigoroso, que eu preciso de um certo cuidado com extravasamento..." (Fabia)

"...Ele é um paciente diferenciado nesse aspecto, que ele vai passar mal, ele vai ter algumas reações que um paciente de clínica não vai ter..." (Fabia)

A quimioterapia é, dentre as modalidades de tratamento, a que possui maior incidência de cura de muitos tumores, incluindo os mais avançados, e a que mais aumenta a sobrevida dos portadores de câncer. É uma modalidade de tratamento sistêmica, em que os agentes antineoplásicos são tóxicos a qualquer tecido de rápida proliferação, normais ou cancerosos, e tem como consequência o aparecimento de efeitos colaterais. Requer atenção, conhecimento e destreza para aplicação e cuidados (BONASSA,2005).

As questões biológicas, como dor e procedimentos invasivos, juntamente com a parte social, como o isolamento e mudanças na rotina, além do medo do desconhecido, geram um desgaste emocional em todos os envolvidos no cuidar (FONSECA; SOARES, 2006).

Ana e Estela citaram como uma das especificidades no cuidado ao paciente oncológico o desgaste emocional do paciente.

"Ele está muito exposto, sente muita dor, e o emocional né, que é extremamente afetado, abalado." (Ana)

"...com o tempo você começa a ver ele piorando, ele se desgastando. Então cuidar de um paciente oncológico é desgastante tanto pra ele como pra gente." (Estela)
Peterson e Carvalho (2011) demonstraram em seu estudo que enfermeiros que trabalham com pacientes oncológicos encontraram dificuldades em lidar comos sentimentos negativos, emanados pela situação, interferindo no cuidado de enfermagem prestado aos pacientes.

Quando falamos de câncer, a morte logo vem associada, desde o diagnóstico, e mesmo durante todo o tratamento, como referiu à enfermeira Isadora:

"... a partir do momento que o paciente sabe que tem câncer.. ele sabe, ele entra num estado depressivo, de tristeza..." (Isadora)

As experiências registradas revelaram que o câncer é associado à morte não somente pelo paciente, mas também pelo próprio profissional que oferecerá a assistência.

"...em certos momentos os cuidados são paliativos, porque não tem muito o que fazer... para cura" (Carla)

O cuidado paliativo é associado a um cuidado humanizado, no sentido de aliviar a dor e o sofrimento do paciente oncológico.

"...De diminuir a dor, de diminuir o sofrimento, de assim... do cuidado assim, mais humanizado..." (Kelly)

"...São pacientes que sentem muita dor, paciente bastante carente...[...] pede droga direto pra dor, o paciente sofre bastante.[...] Ah eu acho que o cuidado para eles é assim... tem que dar muita atenção não deixar sentir dor e dar muita atenção..." (Gabriela)

"...Você está ali pra amenizar a dor do paciente você ajuda a ter uma morte menos traumática..." (Daniela)

A Organização Mundial de Saúde (OMS) compreende como cuidado paliativo a promoção da qualidade de vida e conforto aos pacientes e a família 
diante de uma doença de ameaça de vida, pelo alívio e prevenção dos sintomas e com apoio também das necessidades psicológicas, emocionais e espirituais (CLEMENTE; SANTOS, 2007).

Ao considerar um cliente fora de possibilidades terapêuticas de cura, pode ser vinculado a esse diagnóstico o rotulado de "terminal", com a ideia errônea de que nada mais pode ser feito. Porém, o paciente está vivo, tem necessidades especiais que devem ser atendidas buscando a qualidade de vida. $\mathrm{O}$ cuidado deve ser planejado com objetivo de promover conforto, alívio e controle dos sintomas, suporte espiritual, psicossocial e apoio no luto (SUSAKI; SILVA; POSSARI, 2006).

\section{Conclusão}

A partir dos depoimentos obtidos através desta pesquisa, concluímos que a percepção do cuidar para os enfermeiros entrevistados abordam contextos como humanização, integralidade e atenção à família, ações inerentes ao cuidado sistematizado e com qualidade.

Os dados sugerem a presença de sentimentos como insegurança e impotência, por parte dos enfermeiros, em cuidar de um paciente oncológico, pela associação à morte, ao sofrimento deste paciente, da família, e pela impossibilidade de cura em algumas situações. Tais experiências e vivências repercutem nas emoções dos profissionais que, algumas vezes, acabam se envolvendo emocionalmente, devido aos períodos prolongados de internação e recidivas.

Através deste estudo, observamos que, em relação aos cuidados específicos a essa clientela, há um direcionamento aos cuidados com a terapêutica, aos cuidados paliativos e ao apoio emocional, psicológico junto aos pacientes com câncer e sua família ou cuidador.

Concluímos, então, que há necessidade de um constante aprimoramento e educação contínua de toda a equipe multiprofissional, inclusive na formação acadêmica, sobre o cuidado direcionado ao paciente com câncer, por ser uma doença de grande e crescente incidência e com grandes repercussões no cenário da saúde.

\section{REFERÊNCIAS}

BACKES et al. O Papel do enfermeiro no contexto hospitalar: a visão de profissionais de saúde. Cienc.

Cuid. Saúde, v.7, n 3, p319-326, Jul/Set, 2008.

BARBOSA, L.G., TELLES FILHO, P.C.P.

Conhecimento de Pacientes oncológicos Sobre Quimioterapia. Ver. Cienc. Cuid. Saude. v.7, n 3, p 370-375, Jul/Set, 2008.

\section{BRASIL - CONSELHO FEDERAL DE ENFERMAGEM. Resolução COFEN 358/2009. Brasília, 2009.}

BRASIL. Estimativa 2012: incidência de câncer no país. Rio de Janeiro: INCA, 2009. Disponível em: http://www1.inca.gov.br/estimativa/2010> Acesso em: 17 maio 2012.

BRASIL, Ministério da Saúde. Resolução 466/ 2012. Disponívelem: <http://conselho.saude.gov.br/ resolucoes/2012/Reso466.pdf $>$ Acessado em: 29/ 08/2013.

BONASSA, E.M.A. Conceitos gerais em quimioterapia antineoplásica. In: Bonassa EMA, Santana TR. Enfermagem em terapêutica oncológica. São Paulo: Atheneu; 2005. p. 3-19.

CARVALHO, C.S.U. A Necessária atenção à família do paciente oncológico. Ver. Brasileira de Cancerologia. v.54, n 1, p. 87-96, 2008.

\section{CELICH, K.L.S. Dimensões do processo de} cuidar na enfermagem: Umolhar da enfermeira. Porto Alegre, 2003.

CLEMENTE, R.P.D.S., SANTOS, E. H. A nãoressuscitação do ponto de vista da enfermagem, em uma unidade de cuidados paliativos oncológicos. Rev Brasileira de Cancerologia. v.53, n 2, p 231-236, 2007. 
FONSECA, A. M; SOARES, E. Desgaste emocional: Depoimentos de enfermeiros que atuam no ambiente hospitalar. Rev Rene, v.7, n 1, p 91 97, jan/abril. 2006.

FONTES, C.A.S.; ALVIM, N.A.T. A Relação humana no cuidado de enfermagem junto ao cliente com câncer submetido à terapia antineoplasica. Acta paul. enferm. v.21, n 1, p 77-83, 2007.

MACHADO et al. Integralidade, formação de saúde, educação em saúde e as propostas do SUS: Uma revisão conceitual. Ciênc. saúde coletiva [online]. 2007, v.12, n 2, p 335-342, 2007.

PETERSON A.A.; CARVALHO E. C. Comunicação terapêutica na enfermagem: dificuldades para o cuidar de idosos com câncer. Rev Bras Enferm, v. 64.n.4.p. 692-7, 2011.

SILVEIRA, C.S; ZAGO, M.M.F. Pesquisa brasileira em enfermagem oncológica: uma revisão integrativa. Revista Latino Americana de

Enermagem, v.14, n 4, p 614-9, julho/ago. 2006.

SUSAKI, T.T.; SILVA, M.J.P.; POSSARI, J.F. Identificação das fases do processo de morrer pelos profissionais de Enfermagem. Acta Paul Enferm. v.19,n.2,p.144-9, 2006.

TANNURE, M.C; GONÇALVES, A.M.P.SAE: Sistematização da assistência de enfermagem: Guia Prático. Rio de Janeiro: Guanabara Koogan, 2008.

VENÂNCIO, J.L. Importância da atuação do psicólogo no tratamento de mulheres com câncer de mama. Rev Brasileira de Cancerologia. v.50, n 1, p 55-63, 2004.

\section{VILA, V.S.C. O significado de qualidade de} vida: perspectivas de indivíduos revascularizados e de seus familiares. 2006. $148 \mathrm{f}$. Escola de Enfermagem de Ribeirão Preto, Universidade de São Paulo, Ribeirão Preto, 2006.

ReCEBIDO EM 6/11/2013

ACEITO EM 3/7/2014 